Article

\title{
Lyapunov Functions for State Observers of Dynamic Systems Using Hamilton-Jacobi Inequalities
}

\author{
Angelo Alessandri ${ }^{(D)}$ \\ The Department of Mechanical, Energetics, Management, and Transportation Engineering (DIME), \\ University of Genoa, Via Opera Pia 15, 16145 Genoa, Italy; alessandri@dime.unige.it
}

Received: 5 January 2020; Accepted: 1 February 2020; Published: 6 February 2020

\begin{abstract}
Lyapunov functions enable analyzing the stability of dynamic systems described by ordinary differential equations without finding the solution of such equations. For nonlinear systems, devising a Lyapunov function is not an easy task to solve in general. In this paper, we present an approach to the construction of Lyapunov funtions to prove stability in estimation problems. To this end, we motivate the adoption of input-to-state stability (ISS) to deal with the estimation error involved by state observers in performing state estimation for nonlinear continuous-time systems. Such stability properties are ensured by means of ISS Lyapunov functions that satisfy Hamilton-Jacobi inequalities. Based on this general framework, we focus on observers for polynomial nonlinear systems and the sum-of-squares paradigm to find such Lyapunov functions.
\end{abstract}

Keywords: Lyapunov function; input-to-state stability; Hamilton-Jacobi inequality

\section{Introduction}

The construction of state observers for nonlinear systems is difficult in general when it is required to ensure the property of global stability for the estimation error. Such a proof is obtained by means of the Lyapunov method. Here we address the stability analysis of the error dynamics by using input-to-state stability (ISS) [1,2], where the disturbances are regarded as input, and the role of the state is played by the estimation error incurred by the observer. Moreover, we will address the design of state observers for polynomial nonlinear systems by using the SOS (sum-of-squares) approach $[3,4]$.

The Lyapunov method has been applied in many research areas to analyze the stability of dynamic systems $[5,6]$. To prove that a system is stable in the sense of Lyapunov, a positive definite function of the state variables is shown to decrease along system trajectories. Recently, extensions of the Lyapunov approach to deal with fractional and impulsive systems have been proposed $[2,7,8]$. Indeed, the stability of the estimation error of observers for noise-free systems can be addressed by using Lyapunov functions. However, the so-called ISS Lyapunov functions turn out to be more appropriate, as the effects of both system and measurement noises are treated, while simultaneously dealing with stability in a noise-free setting. An observer with input-to-state stable error dynamics (ISS observer, for short) is prevented to suffer from the occurrence of phenomena of finite escape and hence the use of ISS analysis is considered in various works on estimation for nonlinear systems. First results are reported in $[9,10]$. The connection between ISS and passivity w.r.t measurement disturbances is dealt with in [11]. ISS sampled-data observers are analyzed in [12]. The design of ISS estimators is addressed in [13-16].

Generally speaking, the first results on the construction of observers for nonlinear continuous-time systems are reported in [17-19]. Next, state-space transformations have been studied to obtain a linear dynamics of the estimation in the new coordinates [20-23]. This entails the solution of partial differential equations (see, e.g., [24]) to find the required transformation but makes the stability analysis straightforward. Later, quadratic Lyapuonv functions have been massively adopted to prove global 
stability [25-27]. The literature on nonlinear observers reports various attempts of improvement by relaxing system assumptions and devising less conservative design conditions (see, e.g., [28,29]). Among others, the so-called high-gain observer has become quite popular [30], especially for the purpose of output feedback control [31]. More recently, Lyapunov functionals have been proposed in [32] to prove stability for time-varying high-gain observers in line with the idea of reducing the peaking during the transient $[33,34]$.

The contribution of this paper lies in highlighting the motivations to rely on ISS to both analyze the stability and construct observers for nonlinear systems. Toward this end, we deal with the problem in quite a general nonlinear setting, where the design of observers is reduced to the satisfaction of a Hamilton-Jacobi inequality [35,36]. Specifically, ISS is regarded in terms of $L_{2}$-gain to measure the attenuation of the estimation error w.r.t. the disturbances. Based on the aforesaid, we focus on the construction of observers for a class of polynomial nonlinear systems by relying on the practical $L_{2}$-gain proposed in [37] and using the sum-of-squares (SOS) approach, which has become widely used in a number of applications involving polynomials [3,4] and is based on semidefinite programming (SDP) [38,39]. Following this approach, we can design observers for such polynomial systems under feasible conditions that can be easily treated.

The paper is organized as follows. The use of ISS to analyze the stability of the estimation error provided by state observers is considered in Section 2. The special case of observers for polynomial systems is discussed in Section 3. Numerical results concerning a case study are presented in Section 4 . The conclusions are drawn in Section 5.

The following notation and definitions will be used throughout the paper. $\mathbb{R}_{\geq 0}$ denotes the set of the nonnegative real numbers. $M_{n \times m}(\mathbb{R})$ stands for the set of the real matrices with $n$ rows and $m$ columns. Given a symmetric $P \in M_{n \times n}(\mathbb{R}), P>0(P<0)$ means that $P$ is positive (negative) definite. Given a continuously differentiable function $f: \mathbb{R}^{n} \times \mathbb{R}^{m} \rightarrow \mathbb{R}$, let us define the gradients $\nabla_{x} f(x, y):=$ $\left(\frac{\partial f(x, y)}{\partial x_{1}} \cdots \frac{\partial f(x, y)}{\partial x_{n}}\right)$ and $\nabla_{y} f(x, y):=\left(\frac{\partial f(x, y)}{\partial y_{1}} \cdots \frac{\partial f(x, y)}{\partial y_{m}}\right)$ as column vectors. The Euclidean norm in $\mathbb{R}^{n}$ is denoted by $|\cdot|$. Let us denote by $B_{r}(c):=\left\{x \in \mathbb{R}^{n}:|x-c| \leq r\right\}$ the closed ball centered in $c \in \mathbb{R}^{n}$ with radius $r>0$. A continuous function $\alpha: \mathbb{R}_{\geq 0} \rightarrow \mathbb{R}_{\geq 0}$ is positive definite if it is null only in zero and is said to belong to class $\mathcal{K}$ if it is strictly increasing. Moreover, it is said to belong to class $\mathcal{K}_{\infty}$ if it is of class $\mathcal{K}$ and also $\lim _{r \rightarrow+\infty} \alpha(r)=+\infty$. A continuous function $\beta: \mathbb{R}_{\geq 0} \times \mathbb{R}_{\geq 0} \rightarrow \mathbb{R}_{\geq 0}$ is said to belong to class $\mathcal{K} \mathcal{L}$ if, for each fixed $s$, the mapping $\beta(r, s)$ belongs to class $\mathcal{K}$ w.r.t. to $r$ and, for each fixed $r$, the mapping $\beta(r, s)$ is decreasing with respect to $s$ and $\lim _{s \rightarrow+\infty} \beta(r, s)=0$. $\mathbb{R}[x]$ denotes the ring of real polynomials of $x=\left(x_{1}, \ldots, x_{n}\right) \in \mathbb{R}^{n} ; \Sigma[x]$ denotes the set of the SOS polynomials of $x$, i.e., of the polynomials written as $p(x)=\sum_{i=1}^{n} f_{i}(x)^{2}$ with $f_{1}(x), \ldots, f_{n}(x) \in \mathbb{R}[x]$. Let us denote by $\mathbb{R}[x]^{p \times q}$ and $\Sigma[x]^{m}$ the ring of real matrix polynomials of size $p \times q$ and the set of the SOS polynomials of degree equal or less than $m$, respectively.

\section{Input-to-State Stability for State Observers}

Estimation for nonlinear noise-free continuous-time systems is usually accomplished by using observers, which are dynamic systems that aim at tracking the state variables by using only incomplete information on the state. Let the dynamic equations be given by

$$
\left\{\begin{array}{l}
\dot{x}=f(x, u) \\
y=h(x)
\end{array}, t \geq 0\right.
$$

where $f: \mathbb{R}^{n} \times \mathbb{R}^{p} \rightarrow \mathbb{R}^{n}$ and $h: \mathbb{R}^{n} \rightarrow \mathbb{R}^{m}$ are smooth functions; $x(t) \in \mathbb{R}^{n}, u(t) \in \mathbb{R}^{p}$, and $y(t) \in$ $\mathbb{R}^{m}$ are the state, input, and measurement vectors, respectively. A full-order state observer is in general described by the dynamic equation

$$
\dot{\hat{x}}=\gamma(\hat{x}, y, u) t \geq 0
$$


where $\hat{x}(t) \in \mathbb{R}^{n}$ is the estimate of $x(t)$ at time $t$ and $\gamma: \mathbb{R}^{n} \times \mathbb{R}^{m} \times \mathbb{R}^{p} \rightarrow \mathbb{R}^{n}$ is a smooth function to be chosen in such a way as to ensure that the estimation error $e:=x-\hat{x}$ with dynamics given by

$$
\dot{e}=f(x, u)-\gamma(x-e, h(x))=: F_{\gamma}(e, x, u)
$$

is asymptotically stable to zero. Moreover, usually $m<n$, i.e., roughly speaking, not all of the state variables are accessible. Notice that the dynamics of the estimation error (3) depends in general on the system trajectory and it would always be preferable to ensure the global asymptotic stability to zero for the estimation error. In line with [11], the interest concerns observers for systems described by (1) with globally asymptotically stable estimation error by finding a suitable mapping $\gamma$ and a smooth Lyapunov function $(e, x) \mapsto V(e, x)$ such that

$$
\begin{aligned}
& \alpha_{1}(|e|) \leq V(e, x) \leq \alpha_{2}(|e|) \\
& \nabla_{e} V \cdot F_{\gamma}(e, x, u)+\nabla_{x} V \cdot f(x, u) \leq-\alpha_{3}(|e|)
\end{aligned}
$$

for all $t \geq 0, e \in \mathbb{R}^{n}$, and $x \in \mathbb{R}^{n}$, where $\alpha_{1}, \alpha_{2}$ of class $\mathcal{K}_{\infty}$ and $\alpha_{3}$ is continuous positive definite.

If the system is affected by disturbances, the performances deteriorate in such a way as to make the estimation error increase with the growth of the uncertainty. Anyway, it is expected to keep the estimation error asymptotically stable to zero without noise. This combination can be given with mathematical rigor by using the notion of ISS. Thus, we focus on

$$
\left\{\begin{array}{l}
\dot{x}=f(x, u)+g(x) w \\
y=h(x)+k(x) w
\end{array} \quad t \geq 0\right.
$$

where $w(t) \in \mathbb{R}^{p}$ is the disturbance; $g: \mathbb{R}^{n} \rightarrow M_{n \times p}(\mathbb{R})$ and $k: \mathbb{R}^{n} \rightarrow M_{m \times p}(\mathbb{R})$ are smooth. Therefore, the dynamics of the estimation error is given by

$$
\dot{e}=f(x, u)+g(x) w-\gamma(x-e, h(x)+k(x) w)=: F_{\gamma}(e, x, u, w)
$$

where $F_{\gamma}(e, x, u, w)$ is used instead of $F_{\gamma}(e, x, u)$ as in equation (3) with a little abuse of notation. Based on the aforesaid, we say that the observer (2) is ISS if there exists a function $\beta$ of class $\mathcal{K} \mathcal{L}$ and a function $\chi$ of class $\mathcal{K}_{\infty}$ such that

$$
|e(t)| \leq \beta(|e(0)|, t)+\chi\left(|w|_{\infty(0, t)}\right) t \geq 0
$$

where $|w|_{\infty(0, t)}:=\underset{0 \leq \tau \leq t}{\operatorname{ess} \sup _{0 \leq t}}|w(\tau)|$. The above ISS statement can be equivalently expressed by means of an ISS Lyapunov function [1]: inequality (8) holds if and only if there exist functions $\alpha_{1}, \alpha_{2}$ of class $\mathcal{K}_{\infty}$ and $\alpha_{3}, \eta$ of class $\mathcal{K}$ such that

$$
\begin{aligned}
& \alpha_{1}(|e|) \leq V(e, x) \leq \alpha_{2}(|e|) \\
& \nabla_{e} V \cdot F_{\gamma}(e, x, u, w)+\nabla_{x} V \cdot f(x, u) \leq-\alpha_{3}(|e|) \quad \text { if } \quad|e| \geq \eta(|w|)
\end{aligned}
$$

for all $t \geq 0, e \in \mathbb{R}^{n}, w \in \mathbb{R}^{q}, x \in \mathbb{R}^{n}$, and $u \in \mathbb{R}^{p}$. The observer problem needs to be more reliably formulated in an ISS framework since a system may admit a Lyapuonv function in a noise-free case, while being not input-to-state stable. By contrast, an input-to-state stable system is asymptotically stable to zero if the external input is null. As pointed out in [11], small noises can cause the instability of the estimation error and thus an observer with a globally asymptotically stable error may exist in the absence of disturbances, whereas the error dynamics turns out to be not input-to-state stable, as shown the following example presented in [15]. 
Example 1. Considering for the system (Example 3, p. 50, [15])

$$
\left\{\begin{array}{l}
\dot{x}_{1}=-x_{1}+2 \\
\dot{x}_{2}=x_{1} x_{3} \\
\dot{x}_{2}=-x_{1} x_{2}+u \\
y_{1}=x_{1}+w_{1} \\
y_{2}=x_{2}+w_{2}
\end{array}\right.
$$

with $u(t)=\sin (t)$, the observer

$$
\left\{\begin{array}{l}
\dot{\hat{x}}_{1}=-\hat{x}_{1}+2+y_{1}-\hat{x}_{1} \\
\dot{\hat{x}}_{2}=\hat{x}_{1} \hat{x}_{3}+y_{1}\left(y_{2}-\hat{x}_{2}\right) \\
\dot{x}_{3}=-\hat{x}_{1} \hat{x}_{2}+u+y_{1}\left(y_{2}-\hat{x}_{2}\right)
\end{array}\right.
$$

provides an estimation error that is asymptotically stable to zero if $w_{1}(t)=w_{2}(t)=0$ for all $t \geq 0$. If, instead, we chose $x_{1}(0)=1, w_{1}(t)=-x_{1}(t)$, and $w_{2}(t)=0$ for all $t \geq 0$, it follows that

$$
\lim _{t \rightarrow+\infty} w_{1}(t)=-2 \quad \lim _{t \rightarrow+\infty} w_{2}(t)=0
$$

and the state is bounded, but the dynamics of the observer turns out to be with $\hat{x}_{1}(t)=1$ for all $t \geq 0$ and

$$
\left\{\begin{array}{l}
\dot{\hat{x}}_{2}=\hat{x}_{3} \\
\dot{\hat{x}}_{3}=-\hat{x}_{2}+\sin (t)
\end{array}\right.
$$

and hence the second and third observer state variables are divergent, thus showing that ISS for the estimation error does not hold since the estimation error is unbounded with a bounded disturbance.

The above example suggests constructing observers together with an ISS Lyapunov function for the dynamics of the associated estimation error. Toward this end, instead of the condition (10), let us consider the equivalent inequality

$$
\nabla_{e} V \cdot F_{\gamma}(e, x, u, w)+\nabla_{x} V \cdot f(x, u) \leq-\alpha_{3}(|e|)+\alpha_{4}(|w|)
$$

where $\alpha_{3}, \alpha_{4}$ are of class $\mathcal{K}_{\infty}$. Once the ISS Lyapunov functions are chosen, one may analyze the effect of attenuation and the disturbances on the estimation error. Thus, using (13), let us consider

$$
V(e(t), x(t))-V\left(e\left(t_{0}\right), x\left(t_{0}\right)\right) \leq-\int_{t_{0}}^{t} \alpha_{3}(|e(s)|) d s+\int_{t_{0}}^{t} \alpha_{4}(|w(s)|) d s
$$

for all $t \geq t_{0} \geq 0$, which provides an upper bound on $V(e(t), x(t))$ based on the initial conditions and a measure of the "energy" of the noises. If $V$ is a continuously differentiable function, (14) holds if (13) is satisfied. The function $V$ is thus a storage function, while $\alpha_{3}$ and $\alpha_{4}$ have to be regarded as dissipation and supply rates, respectively. If $w(\cdot)$ and $e(\cdot)$ belong to the $L_{2}$ space of functions of time, the link between ISS and the $H_{\infty}$ approach is easily found [40]. Let us now focus on the case with the dynamics of the estimation error affine in the noises.

Theorem 1. Consider observer (2) for systems described by (6) such that

$$
F_{\gamma}(e, x, u, w)=H_{\gamma}(e, x, u)+G_{\gamma}(e, x) w
$$


where $H_{\gamma}: \mathbb{R}^{2 n} \rightarrow \mathbb{R}^{n}$ and $G_{\gamma}: \mathbb{R}^{2 n} \times \rightarrow M_{n \times p}(\mathbb{R})$. If there exists a continuously differentiable function $(e, x) \mapsto V(e, x)$ such that the inequalities (4) are satisfied for some functions $\alpha_{1}, \alpha_{2}$ of class $\mathcal{K}_{\infty}$ and the Hamilton-Jacobi inequality

$$
\nabla_{e} V \cdot H_{\gamma}(e, x)+\frac{\left|\nabla_{e} V \cdot G_{\gamma}(e, x)+\nabla_{x} V \cdot g(x)\right|^{2}}{2 \mu^{2}}+\nabla_{x} V \cdot f(x, u)+\frac{|e|^{2}}{2} \leq 0
$$

holds for some $\mu>0$ and all $t \geq 0, x \in \mathbb{R}^{n}, u \in \mathbb{R}^{p}$, and $e \in \mathbb{R}^{n}$. Then, $V(e, x)$ is an ISS Lyapunov function and the $L_{2}-t o-L_{2}$ dissipative inequality (14) is satisfied with $\alpha_{3}(r)=\frac{1}{2} r^{2}, \alpha_{4}(r)=\frac{\mu^{2}}{2} r^{2}$, i.e.,

$$
V(e(t), x(t))-V\left(e\left(t_{0}\right), x\left(t_{0}\right)\right) \leq-\frac{1}{2} \int_{t_{0}}^{t}|e(s)|^{2} d s+\frac{\mu^{2}}{2} \int_{t_{0}}^{t}|w(s)|^{2} d s
$$

for all $t \geq t_{0} \geq 0$ and thus observer (2) is input-to-state stable.

Proof. From Equations (7) and (15), we obtain

$$
\begin{aligned}
\frac{\mathrm{d}}{\mathrm{d} t} V(e, x) & =\nabla_{e} V \cdot H_{\gamma}+\left(\nabla_{e} V \cdot G_{\gamma}+\nabla_{x} V \cdot g\right) w+\nabla_{x} V \cdot f \\
& =\nabla_{e} V \cdot H_{\gamma}+\frac{\mu^{2}}{2}\left(\frac{2\left(\nabla_{e} V \cdot G_{\gamma}+\nabla_{x} V \cdot g\right) w}{\mu^{2}}\right)+\nabla_{x} V \cdot f \\
& =\nabla_{e} V \cdot H_{\gamma}+\frac{\mu^{2}}{2}\left(-|w|^{2}+\frac{2\left(\nabla_{e} V \cdot G_{\gamma}+\nabla_{x} V \cdot g\right) w}{\mu^{2}}-\frac{\left|\nabla_{e} V \cdot G_{\gamma}+\nabla_{x} V \cdot g\right|^{2}}{\mu^{4}}\right. \\
& \left.+\frac{\left|\nabla_{e} V \cdot G_{\gamma}+\nabla_{x} V \cdot g\right|^{2}}{\mu^{4}}+|w|^{2}\right)+\nabla_{x} V \cdot f \\
& =\nabla_{e} V \cdot H_{\gamma}-\frac{\mu^{2}}{2}\left|w-\frac{\nabla_{e} V \cdot G_{\gamma}+\nabla_{x} V \cdot g}{\mu^{2}}\right|^{2}+\frac{\left|\nabla_{e} V \cdot G_{\gamma}+\nabla_{x} V \cdot g\right|^{2}}{2 \mu^{2}}+\nabla_{x} V \cdot f+\frac{\mu^{2}}{2}|w|^{2} \\
& \leq \nabla_{e} V \cdot H_{\gamma}+\frac{\left|\nabla_{e} V \cdot G_{\gamma}+\nabla_{x} V \cdot g\right|^{2}}{2 \mu^{2}}+\nabla_{x} V \cdot f+\frac{\mu^{2}}{2}|w|^{2} .
\end{aligned}
$$

Using the assumption (16), the previous inequality yields

$$
\frac{\mathrm{d}}{\mathrm{d} t} V(e, x) \leq-\frac{1}{2}|e|^{2}+\frac{\mu^{2}}{2}|w|^{2}
$$

and hence we can conclude about ISS with inequality (17) as a final result.

We say that the observer admits an $L_{2}$-gain $\mu$ w.r.t. $w$ if (17) holds. Clearly, a small gain is preferable since it entails a stronger noise attenuation. In the following, we will analyze how to take care of the Hamilton-Jacobi inequality (16), starting with the following example.

Example 2. Let us consider

$$
\left\{\begin{array}{l}
\dot{x}_{1}=x_{1}+\sin \left(x_{2}\right) w \\
\dot{x}_{2}=x_{1}-2 x_{2}+\exp \left(-x_{2}\right) \\
y=x_{1}+x_{2}
\end{array}\right.
$$

and let

$$
\left\{\begin{array}{l}
\dot{\hat{x}}_{1}=\hat{x}_{1}+2\left(y-\hat{x}_{1}-\hat{x}_{2}\right) \\
\dot{\hat{x}}_{2}=\hat{x}_{1}-2 \hat{x}_{2}+\exp \left(-\hat{x}_{2}\right)-\left(y-\hat{x}_{1}-\hat{x}_{2}\right)
\end{array}\right.
$$

be a candidate observer for system (19). It is straightforward to obtain

$$
\dot{e}=H(e, x)+G(e, x) w
$$


where

$$
\begin{aligned}
& H(e, x)=\left(\begin{array}{c}
-e_{1}-2 e_{2} \\
2 e_{1}-e_{2}+\exp \left(-x_{2}\right)\left(1-\exp \left(e_{2}\right)\right)
\end{array}\right) \\
& G(e, x)=\left(\begin{array}{c}
\sin \left(x_{2}\right) \\
0
\end{array}\right) .
\end{aligned}
$$

Let us consider $V(e)=\frac{1}{2} e_{1}^{2}+\frac{1}{2} e_{2}^{2}$ as a candidate ISS Lyapunov function. We have

$$
\nabla_{e} V \cdot H+\frac{\left(\nabla_{e} V \cdot G\right)^{2}}{2 \mu^{2}}+\frac{|e|^{2}}{2}=-\frac{1}{2} e_{1}^{2}-\frac{1}{2} e_{2}^{2}+\exp \left(-x_{2}\right)\left(1-\exp \left(e_{2}\right)\right) e_{2}+\frac{\sin \left(x_{2}\right)^{2}}{2 \mu^{2}} e_{1}^{2} .
$$

Since $\exp \left(-x_{2}\right)\left(1-\exp \left(e_{2}\right)\right) e_{2} \leq 0, \forall e_{2}, x_{2} \in \mathbb{R}$, and $\sin \left(x_{2}\right)^{2} \leq 1, \forall x_{2} \in \mathbb{R}$, Equation (21) yields

$$
\nabla_{e} V \cdot H+\frac{\left(\nabla_{e} V \cdot G\right)^{2}}{2 \mu^{2}}+\frac{|e|^{2}}{2}=-\frac{1}{2}\left(1-\frac{1}{\mu^{2}}\right) e_{1}^{2}-\frac{1}{2} e_{2}^{2}<0
$$

for $e \neq 0$ if $\mu>1$. Thus, observer (20) is ISS with an $L_{2}$-gain larger than 1.

The next section regards the application of what has been presented so far to observers for a class of polynomial systems.

\section{State Observers for Polynomial Systems}

After choosing a given observer structure, the problem reduces to find an ISS Lyapunov function that the condition (16) holds. Unfortunately, conditions like (16) are not easy to be satisfied, which has motivated the investigation on the weaker notion of practical $L_{2}$-gain proposed in [37]. We will address the problem of constructing observers with practical $L_{2}$-gain for a class of polynomial systems.

Let us focus on

$$
\left\{\begin{array}{l}
\dot{x}=A x+f(x)+g(x) w \\
y=C x+k(x) w
\end{array}, t \geq 0\right.
$$

where $A \in \mathbb{R}^{n \times n}, C \in \mathbb{R}^{m \times n}, f(x) \in \mathbb{R}[x]^{n \times n}, g(x) \in \mathbb{R}[x]^{n \times p}, k(x) \in \mathbb{R}[x]^{m \times p}$, and the pair $(A, C)$ is detectable. To estimate the state, we rely on observers given by

$$
\dot{\hat{x}}=A \hat{x}+f(\hat{x})+L(y-C \hat{x}), t \geq 0
$$

with the gain $L \in \mathbb{R}^{n \times m}$ chosen such that $A-L C$ has eigenvalues with a strictly negative real part. This condition can be satisfied owing to the detectability assumption and ensures that the estimation error dynamics

$$
\dot{e}=(A-L C) e+f(x)-f(\hat{x})+(g(x)-L k(x)) w
$$

is locally asymptotically stable to zero in the absence of noise, i.e., with $w(t) \equiv 0$ for all $t \geq 0$. More specifically, in such a case we have

$$
\dot{e}=(A-L C) e+f(x)-f(x-e) .
$$

In the noisy setting, for the sake of brevity, let us rewrite Equation (24) as

$$
\dot{e}=H(e, x, L)+G(x, L) w
$$

with

$$
H(e, x, L):=(A-L C) e+f(x)-f(x-e) \in \mathbb{R}[x, e]^{n \times 1} \quad G(x, L):=g(x)-L k(x) \in \mathbb{R}[x]^{n \times p} .
$$


Though a polynomial system may be asymptotically stable without admitting a polynomial Lyapunov function [41], it is convenient to search for polynomial ISS Lyapunov functions $V(e, x)$ and especially such that inequality (17) holds for some $\mu>0$. Given a candidate Lyapunov function, we may apply the SOS decomposition of such a function and of the opposite of its time derivative by using a positivity certification, which does not depend on the characteristics of the chosen polynomial since the following holds $[39,42]$.

Theorem 2. A polynomial $p(e) \in \mathbb{R}[e]^{2 d}$ in $e=\left(e_{1}, \ldots, e_{n}\right) \in \mathbb{R}^{n}$ has sum-of-squares decomposition (or is said to be SOS) if and only if there exists a real symmetric and positive semidefinite matrix $P \in \mathbb{R}^{s(d) \times s(d)}$ such that $p(e)=v_{d}(e)^{\top} P v_{d}(e) \in \Sigma[e]$, where $v_{d}(e)$ is the vector of all the monomials in the components of $e \in \mathbb{R}^{n}$ of degree equal to or less than $d \in \mathbb{N}$, i.e.,

$$
v_{d}(e):=\left(1, e_{1}, \ldots, e_{n}, e_{1} e_{2}, \ldots, e_{n-1} e_{n}, e_{n}^{2}, \ldots, e_{1}^{d}, \ldots, e_{n}^{d}\right)
$$

of dimension

$$
s(d):=\left(\begin{array}{c}
n+d \\
d
\end{array}\right)
$$

Proof. See (Proposition 2.1, p. 17, [43]).

Thus, from now on, we will refer to well-established definitions as follows [44]. A polynomial $p(e) \in \mathbb{R}[e]^{2 d}$ is $\varepsilon$-SOS polynomial if $p(e)-\varepsilon \sum_{i=1}^{n} e_{i}^{2} \in \Sigma[e]^{2 d}$ with $d \in \mathbb{N}, d \geq 1$ is SOS for some "small" tolerance $\varepsilon>0$. In addition, a polynomial, square matrix $M(x)$ with $M_{i j}(x) \in \mathbb{R}[x]$ for $i, j=1, \ldots, s(d)$ is said to be an $\varepsilon$-SOS matrix if the bipartite polynomial $p(e, x):=v_{d}(e)^{\top} M(x) v_{d}(e)$ is $\varepsilon$-SOS for all $x \in \mathbb{R}^{n}$. In this respect, $p(e, x)$ is said to be a bipartite $\varepsilon$-SOS polynomial.

Theorem 3. Consider observer (23) for system (22). If there exist a bipartite $\varepsilon-S O S V(e, x)$ and $\mu>0$ such that

$$
\begin{aligned}
& \eta(e, x)^{\top} \Pi_{e}^{\top} H(e, x, L)+\eta(e, x)^{\top} \Pi_{x}^{\top}(A x+f(x))+|\eta(e, x)|^{2}+\frac{|e|^{2}}{2} \leq 0 \\
& \left(\begin{array}{cc}
2 I & \Pi_{e}^{\top} G(x, L)+\Pi_{x}^{\top} g(x) \\
G(x, L)^{\top} \Pi_{e}+g(x)^{\top} \Pi_{x} & \mu^{2} I
\end{array}\right)>0
\end{aligned}
$$

for all $x \in \mathbb{R}^{n}$ and $e \in \mathbb{R}^{n}$, where $\eta(e, x) \in \mathbb{R}^{q}$ is the column vector of all the monomials in $e$ and $x$ appearing in $\nabla_{e} V(e, x)$ and $\nabla_{x} V(e, x)$ with $\Pi_{e}, \Pi_{x} \in \mathbb{R}^{n \times q}$ such that

$$
\nabla_{e} V(e, x)=\Pi_{e} \eta(e, x) \quad \nabla_{x} V(e, x)=\Pi_{x} \eta(e, x) .
$$

Then, observer (23) is ISS with $L_{2}$-gain equal to $\mu$.

Proof. The proof is line with [14]. The time derivative of $V(e, x)$ is given by

$$
\begin{aligned}
& \frac{\mathrm{d}}{\mathrm{d} t} V(e, x)=\nabla_{e} V(e, x) \cdot H(e, x, L)+\nabla_{e} V(e, x) \cdot(G(x, L) w)+\nabla_{x} V(e, x) \cdot(A x+f(x)) \\
& +\nabla_{x} V(e, x) \cdot(g(x) w)
\end{aligned}
$$

and, using (27), we obtain

$$
\frac{\mathrm{d}}{\mathrm{d} t} V(e, x)=\eta(e, x)^{\top} \Pi_{e}^{\top} H(e, x, L)+\eta(e, x)^{\top} \Pi_{x}^{\top}(A x+f(x))+\eta(e, x)^{\top}\left(\Pi_{e}^{\top} G(x, L)+\Pi_{x}^{\top} g(x)\right) w
$$

Using (Lemma 4.2, p. 861 in [14]), from (26), it follows that

$$
\eta(e, x)^{\top}\left(\Pi_{e}^{\top} G(x, L)+\Pi_{x}^{\top} g(x)\right) w \leq \eta(e, x)^{\top} \eta(e, x)+\frac{\mu^{2}}{2} w^{\top} w .
$$


and, after replacing this upper bound in Equation (28), it follows that (18) (see the proof of Theorem 1) holds if (25) is satisfied, which allows for concluding.

Based on the aforesaid, one can proceed with the observer design. Let be $d \in \mathbb{N}$ with $d \geq 1$ and $\varepsilon>0$, and the problem to solve is the following.

Problem 1. Find a bipartite $\varepsilon$-SOS $V(e, x), L \in \mathbb{R}^{n \times m}$, and $\mu \geq 0$ such that the conditions (25) and (26) hold and $\mu$ is minimized.

Indeed, the available SOS tools do not allow for solving Problem 1. Thus, we will address the observer design by relying on the so-called practical $L_{2}$-gain proposed in [37]. However, first of all consider the pure linear case by dealing with a system like in (22) without $f(x)$ and with $g(x)$ and $k(x)$ being real matrices, as follows:

$$
\left\{\begin{array}{l}
\dot{x}=A x+D w \\
y=C x+E w
\end{array} \quad t \geq 0\right.
$$

where the pair $(A, C)$ is detectable, $D \in \mathbb{R}^{n \times p}$, and $E \in \mathbb{R}^{m \times p}$. The observer equation is as follows:

$$
\dot{\hat{x}}=A \hat{x}+L(y-C \hat{x}) t \geq 0 .
$$

Since the dynamics of the estimation error is

$$
\dot{e}=(A-L C) e+(D-L E) w
$$

and, after a little algebra and using the Schur complement (p. 39, [45]), inequality (16) becomes

$$
\left(\begin{array}{cc}
A P-C^{\top} Y^{\top}+P A-Y C+\frac{I}{2} & P D-Y E \\
(P D-Y E)^{\top} & -\frac{\mu^{2}}{2} I
\end{array}\right)<0
$$

with $P=P^{\top} \in \mathbb{R}^{n \times n}, P>0$ and $Y=P L \in \mathbb{R}^{n \times m}$. Since the pair $(A, C)$ is detectable, the above linear matrix inequality (LMI) admits a solution in $P$ and $Y$, and it follows $L=P^{-1} Y$. Moreover, using popular LMI-based tools [45], it is straightforward to minimize $\mu^{2}$ exactly since the problem is convex. Thus, one may search for approximate solutions to Problem 1 for Equations (22) and (23) by relying on the solution obtained after neglecting the nonlinearities. Thus, instead of Problem 1, let us consider the much simpler LMI problem

$$
\begin{aligned}
& \min \delta \quad \text { w.r.t. } \quad Y, P>0, \delta>0 \\
& \left(\begin{array}{cc}
A P-C^{\top} Y^{\top}+P A-Y C+\frac{I}{2} & P D-Y E \\
(P D-Y E)^{\top} & -\frac{\delta}{2} I
\end{array}\right)<0 .
\end{aligned}
$$

After finding the solution denoted by $Y^{*}, P^{*}, \delta^{*}$, the gain is given by $L^{*}=\left(P^{*}\right)^{-1} Y^{*}$, which provides a local $L_{2}$-gain $\mu=\sqrt{\delta^{*}}$ with the Lyapunov function $V(e)=e^{\top} P^{*} e$ for Equations (22) and (23). Since it is difficult to ensure a constant $L_{2}$-gain over all the operating points, we will rely on the notion of practical $L_{2}$-gain proposed in [37]. Based on the practical $L_{2}$-gain, one can overcome the problem of finding a globally fixed $L_{2}$-gain. More specifically, assume that there exists a bipartite $\varepsilon$-SOS function $V(e, x)$ such that

$$
\nabla_{e} V \cdot H\left(e, x, L^{*}\right)+\nabla_{x} V \cdot(A x+f(x))+\frac{|e|^{2}}{2}<0
$$

for $e \neq 0$, then

$$
\nabla_{e} V \cdot H\left(e, x, L^{*}\right)+\frac{\left|\nabla_{e} V \cdot G\left(x, L^{*}\right)+\nabla_{x} V \cdot g(x)\right|^{2}}{2 \mu(e, x)^{2}}+\nabla_{x} V \cdot(A x+f(x))+\frac{|e|^{2}}{2} \leq 0
$$


holds if $(e, x) \mapsto \mu(e, x): \mathbb{R}^{n} \times \mathbb{R}^{n} \rightarrow \mathbb{R}_{\geq 0}$ is chosen such that

$$
\mu(e, x) \geq \mu(e, x)^{*}:=\frac{\left|\nabla_{e} V \cdot G\left(x, L^{*}\right)+\nabla_{x} V \cdot g(x)\right|}{\sqrt{-2 \nabla_{e} V \cdot H\left(e, x, L^{*}\right)-2 \nabla_{x} V \cdot(A x+f(x))-|e|^{2}}}
$$

and thus $\mu(e, x)^{*}$ is referred to as practical $L_{2}$-gain. Based on the aforesaid, the problem to solve reduces to the following.

Problem 2. Given $L^{*} \in \mathbb{R}^{n \times m}$ as a solution of (29), find a bipartite $\varepsilon$-SOS $V(e, x)$ such that inequality (30) holds.

The solution of Problem 2 allows for adopting a more flexible notion of attenuation w.r.t. the noises by using an $L_{2}$-gain that may depend on both $e$ and $x$. Following the same reasoning of the proof of Theorem 1, instead of inequality (18), we obtain

$$
\frac{\mathrm{d}}{\mathrm{d} t} V(e, x) \leq-\frac{1}{2}|e|^{2}+\frac{\mu(e, x)^{2}}{2}|w|^{2}
$$

from (31) if inequality (30) holds with $\mu(e, x)$ subject to (32). Thus, the condition (33) may be used whenever the $L_{2}$-gain cannot be bounded from above in the usual sense such as in Example 2, for which the lower bound (given by 1 ) holds globally, i.e., for all $e, x \in \mathbb{R}^{n}$. In practice, the attenuation w.r.t. the disturbances are left to vary in the state space. Moreover, the condition (31) is easier to be satisfied as compared with (30). Indeed, additional constraints can be introduced by constraining the estimation error to belong to suitable compact sets and/or taking into account the boundedness of the state trajectories [14]. This allows for facilitating feasibility when solving Problem 2.

In the next section, we will show numerical results obtained in a simulation case study.

\section{Numerical Results}

In this section, we will show how to apply the proposed approach by exploiting the polynomial structure of the system and observer equations. Thanks to the use of SOS toolbox [44], we will find an ISS polynomial Lyapunov function, which guarantees stability, as illustrated so far. The numerical results are obtained by dealing with the Van der Pol oscillator, which is an interesting example of a polynomial system with a stable limit cycle (p. 57, [5]) and is thus well-suited for the purpose of testing.

Let us consider a system with two coupled Van der Pol oscillators with the first and third state variable as outputs, i.e.,

$$
\left\{\begin{array}{l}
\dot{x}=A x+f(x)+D w \\
y=C x+E w
\end{array}\right.
$$

where $x \in \mathbb{R}^{4}, w \in \mathbb{R}^{4}, y \in \mathbb{R}^{2}$,

$$
\begin{aligned}
& A=\left(\begin{array}{rrrr}
0 & 1 & 0 & 0 \\
-1 & 1 & 1 & 0 \\
0 & 0 & 0 & 1 \\
1 & 0 & -1 & 1
\end{array}\right) \quad f(x)=\left(\begin{array}{c}
0 \\
-x_{1}^{2} x_{2} \\
0 \\
-x_{3}^{2} x_{4}
\end{array}\right) \quad D=\left(\begin{array}{llll}
0 & 0 & 0 & 0 \\
1 & 0 & 0 & 0 \\
0 & 0 & 0 & 0 \\
0 & 1 & 0 & 0
\end{array}\right) \\
& C=\left(\begin{array}{llll}
1 & 0 & 0 & 0 \\
0 & 0 & 1 & 0
\end{array}\right) \quad E=\left(\begin{array}{llll}
0 & 0 & 1 & 0 \\
0 & 0 & 0 & 1
\end{array}\right) .
\end{aligned}
$$

The observer is thus given by

$$
\dot{\hat{x}}=A \hat{x}+f(\hat{x})+L(y-C \hat{x})
$$


with the gain

$$
L=\left(\begin{array}{ll}
3.0189 & 0.2703 \\
3.9558 & 0.6106 \\
0.2703 & 3.0189 \\
0.6106 & 3.9558
\end{array}\right)
$$

obtained by solving problem (29) with Yalmip [46]. The solution provides a value of $\mu$ equal to 4.6575 . Using the SOS toolbox [44], we solved Problem 2 with $\varepsilon=10^{-9}$ and additional constraints $e \in B_{r}(\sqrt{10})$ and $x \in B_{r}(10)$, thus obtaining the Lyapunov function

$$
\begin{aligned}
& V(e, x)=-1.0962 \mathrm{e}-10 e_{1} e_{2} x_{1}^{2}+2.0092 \mathrm{e}-11 e_{1} e_{4} x_{3}^{2}+2.6663 \mathrm{e}-10 e_{2}^{2} x_{1}^{2}+2.6452 \mathrm{e}-12 e_{2} e_{3} x_{1}^{2} \\
& -4.5329 \mathrm{e}-11 e_{2} e_{4} x_{1}^{2}+2.2772 \mathrm{e}-11 e_{2} e_{4} x_{3}^{2}+7.7374 \mathrm{e}-11 e_{3} e_{4} x_{3}^{2}-2.8571 \mathrm{e}-10 e_{4}^{2} x_{3}^{2}+4.3459 \mathrm{e}-6 e_{1}^{2} \\
& -2.0934 \mathrm{e}-6 e_{1} e_{2}-1.3632 \mathrm{e}-7 e_{1} e_{3}-1.6176 \mathrm{e}-7 e_{1} e_{4}+8.6434 \mathrm{e}-7 e_{2}^{2}-1.6176 \mathrm{e}-7 e_{2} e_{3}+3.9843 \mathrm{e}-8 e_{2} e_{4} \\
& +4.3459 \mathrm{e}-6 e_{3}^{2}-2.0934 \mathrm{e}-6 e_{3} e_{4}+8.6435 \mathrm{e}-7 e_{4}^{2}
\end{aligned}
$$

which certifies that this observer is ISS.

Figures 1-4 illustrate the simulation results. Figures 1 and 2 showcase the transient behavior of the state variables and corresponding estimates in two different simulation runs, i.e., in a noise-free case and under the presence of random noises. Such simulation runs show that the time response of the observer is less than the oscillation period of the oscillators, namely it converges quite quickly in such a way as to feed the possible close-loop controllers with the estimates for the purpose of syncronization (see [47] and the references therein). The behaviors at the regime can be analyzed by looking at Figures 3 and 4. In Figure 3, it is shown that the estimation error is kept to zero when the initial state and estimated state coincide and neither system nor measurement disturbances affect the plant. In the presence of noises, the estimation error stays bounded since the estimated state is close to the true state over time, as depicted in Figure 4.
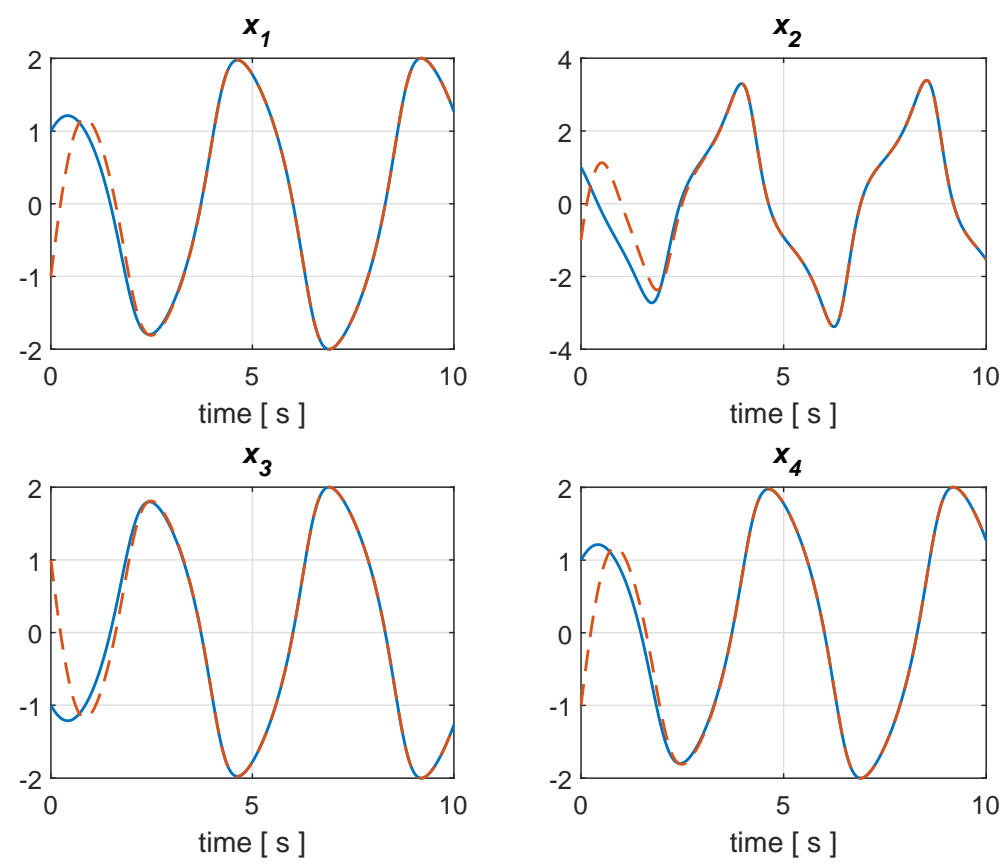

Figure 1. Simulation run without noises and with $x(0)=(1,1,-1,-1)$ and $\hat{x}(0)=(-1,-1,1,1)$, where the state variables are in blue and their estimates are in dashed red. 

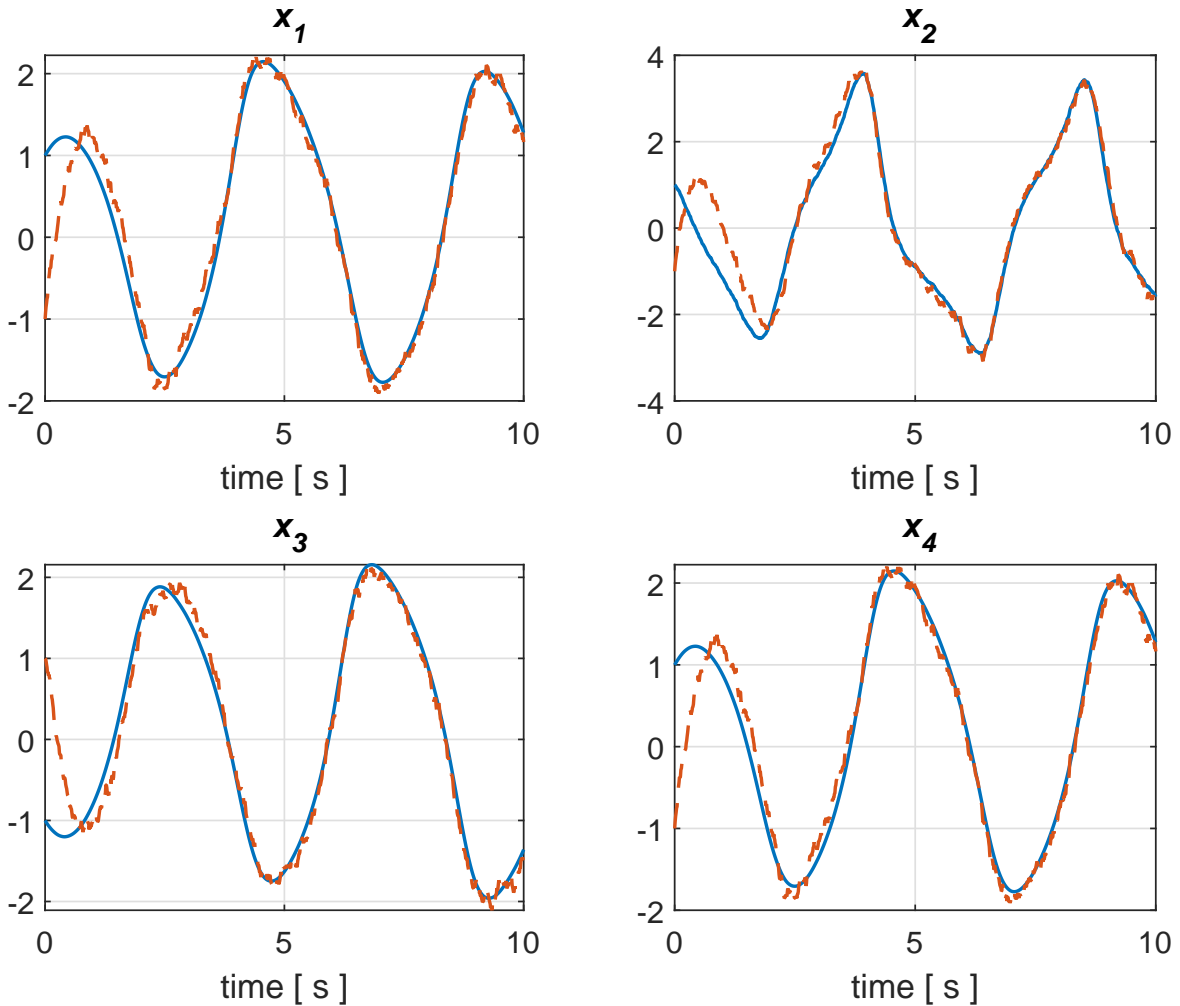

Figure 2. Simulation run with uniformly distributed noises $w_{i}, i=1,2,3,4$, in $[-1,1], x(0)=$ $(1,1,-1,-1)$, and $\hat{x}(0)=(-1,-1,1,1)$, where the state variables are in blue and their estimates are in dashed red.
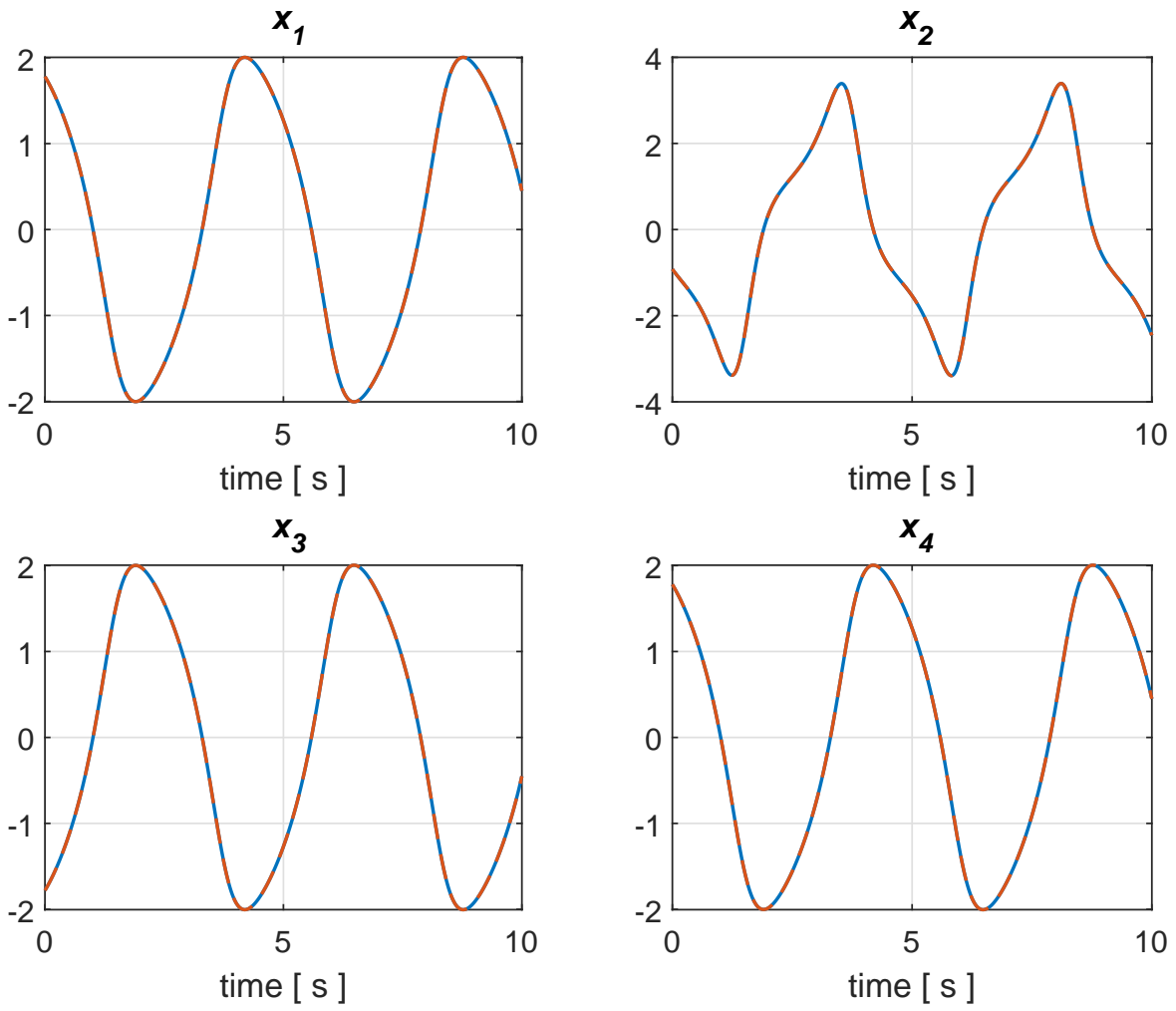

Figure 3. Simulation run at regime without noises and with $x(0)=\hat{x}(0)=$ $(1.7809,-0.9185,-1.7809,1.7809)$, where the state variables are in blue and their estimates are in dashed red. 

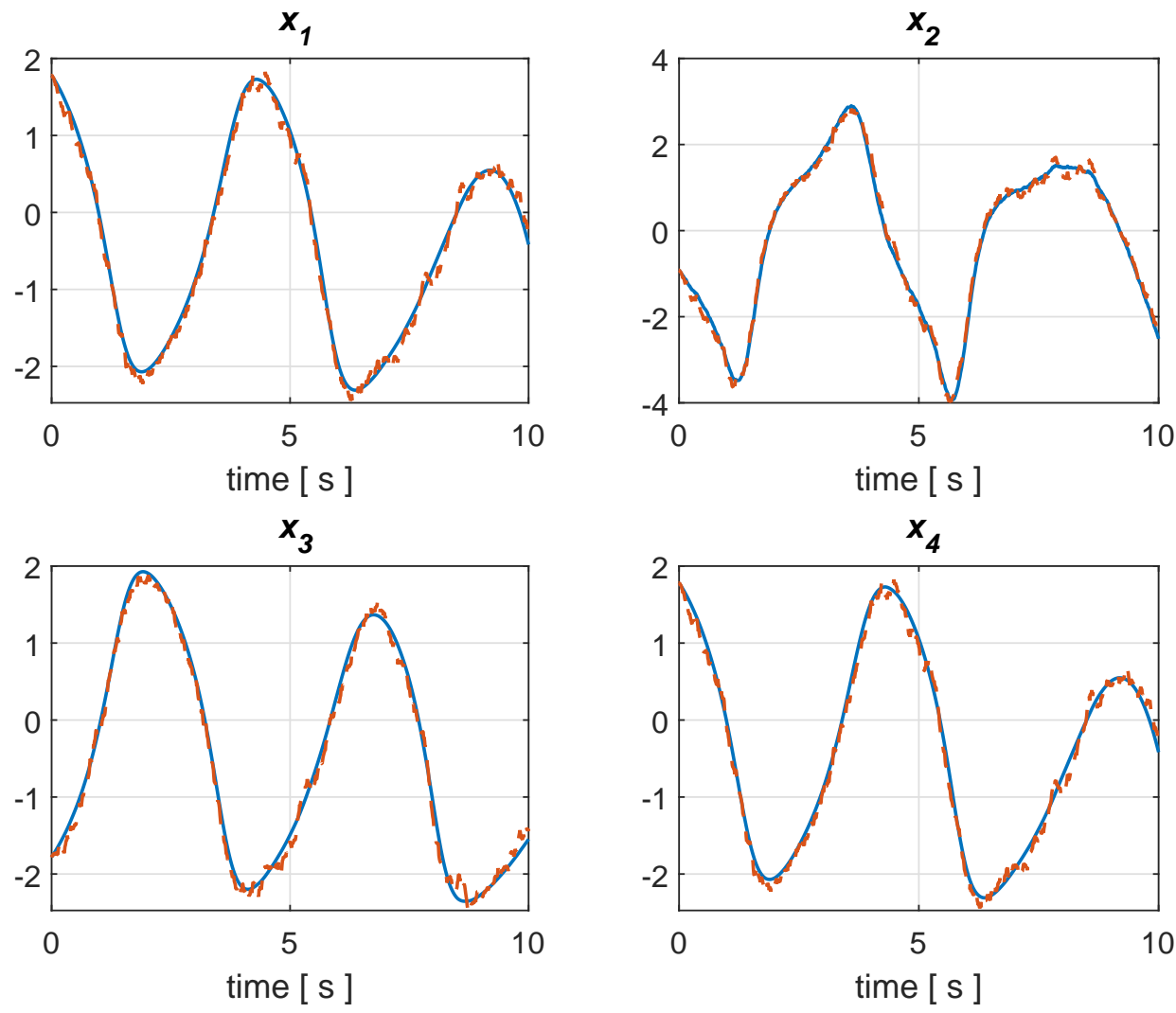

Figure 4. Simulation run at regime with uniformly distributed noises $w_{i}, i=1,2,3,4$, in $[-1,1]$, $x(0)=\hat{x}(0)=(1.7809,-0.9185,-1.7809,1.7809)$, where the state variables are in blue and their estimates are in dashed red.

\section{Conclusions}

In the paper, we have considered the stability issues concerning the estimation error given by state observers, usually employed to estimate the state variables of dynamic systems asymptotically. While the problem is pretty well-studied, only recently research efforts have been devoted to apply ISS, which is shown to be more appropriate as compared with standard Lyapunov stability. In addition, we have addressed the problem of constructing observers with desired performances in terms of $L_{2}$-gain, as shown in more detail with the case study on observers for a class of polynomial systems. Future work will regard the investigation of the proposed approach based on ISS for other kinds of observers such as those considered in $[16,48]$. Another direction of research will be focused on the study of ISS in a discrete-time setting for moving horizon estimation [49].

Funding: This research received no external funding.

Conflicts of Interest: The author declares no conflict of interest.

\section{Abbreviations}

The following abbreviations are used in this manuscript:

ISS input-to-state stability

SDP semidefinite programming

SOS sum-of-squares

\section{References}

1. Sontag, E. The ISS philosophy as a unifying framework for stability-like behavior. In Lecture Notes in Control and Information Sciences; Isidori, A., Lamnabhi-Lagarrigue, F., Eds.; Springer: London, UK, 2000; pp. $443-467$. 
2. Liu, B.; Xu, B.; Zhang, G.; Tong, L. Review of some control theory results on uniform stability of impulsive systems. Mathematics 2019, 7, 1186. [CrossRef]

3. Parrilo, P. Structured Semidefinite Programs and Semialgebraic Geometry Methods in Robustness and Optimization. Ph.D. Thesis, Department of Electrical Engineering, California Institute of Technology, Pasadena, CA, USA, 2000.

4. Parrillo, P. Semidefinite programming relaxations for semialgebraic problems. Math. Program. 2003, 96, 293-320.

5. Khalil, H.K. Nonlinear Systems; Prentice Hall: Upper Saddle River, NJ, USA, 1996.

6. Sontag, E. Mathematical Control Theory: Deterministic Finite Dimensional Systems, 2nd ed.; Springer: Berlin/Heidelberg, Germany, 1998.

7. Wu, G.C.; Baleanu, D.; Luo, W.H. Lyapunov functions for Riemann-Liouville-like fractional difference equations. Appl. Math. Comput. 2017, 314, 228-236. [CrossRef]

8. Wu, G.C.; Baleanu, D. Stability analysis of impulsive fractional difference equations. Fract. Calc. Appl. Anal. 2018, 21, 354-375. [CrossRef]

9. Chaves, M.; Sontag, E. State-estimators for chemical reaction networks of Feinberg-Horn-Jackson zero deficiency type. Eur. J. Control 2002, 8, 343-359.

10. Alessandri, A. Observer design for nonlinear systems by using input-to-state stability. In Proceedings of the 43rd IEEE Conference on Decision and Control, Nassau, Bahamas, 14-17 December 2004; pp. 3892-3897.

11. Shim, H.; Seo, J.; Teel, A. Nonlinear observer design via passivation of the error dynamics. Automatica 2003, 39, 885-892.

12. Karafyllis, I.; Kravaris, C. From continuous-time design to sampled-data design of observers. IEEE Trans. Autom. Control 2009, 54, 2169-2174.

13. Alessandri, A.; Cervellera, C.; Macciò, D.; Sanguineti, M. Optimization based on quasi-Monte Carlo sampling to design state estimators for nonlinear systems. Optimization 2010, 59, 963-984.

14. Rehak, B. Sum-of-squares based observer design for polynomial systems with a known fixed time delay. Kybernetica 2015, 51, 856-873.

15. Shim, H.; Liberzon, D. Nonlinear observers robust to measurement disturbances in an ISS sense. IEEE Trans. Autom. Control 2016, 61, 48-61.

16. Astolfi, D.; Alessandri, A.; Zaccarian, L. Stubborn ISS redesign for nonlinear high-gain observers. In Proceedings of the 20th IFAC World Congress, Toulouse, France, 9-14 July 2017; pp. 15992-15997.

17. Thau, F. Observing the state of nonlinear dynamic systems. Int. J. Control 1973, 17, 471-479.

18. Kou, S.; Elliott, D.; Tarn, T. Exponential observers for nonlinear dynamic systems. Inf. Control 1975, 29, 204-216.

19. Banks, S. A note on nonlinear observers. Int. J. Control 1981, 34, 185-190.

20. Krener, A.; Isidori, A. Linearization by output injection and nonlinear observers. Syst. Control Lett. 1983, 3, 47-52.

21. Bestle, D.; Zeitz, M. Canonical form observer design for nonlinear time-variable systems. Int. J. Control 1983, 38, 419-431.

22. Krener, A.; Respondek, W. Nonlinear observer with linearizable error dynamics. SIAM J. Control Optim. 1985, 23, 197-216.

23. Keller, H. Nonlinear observer design by transformation into a generalized observer canonical form. Int. J. Control 1987, 46, 1915-1930.

24. Kazantzis, N.; Kravaris, C. Nonlinear observer design using Lyapunov's auxiliary theorem. Syst. Control Lett. 1998, 34, 241-247.

25. Walcott, B.; Zak, S. State observation of nonlinear uncertain dynamical systems. IEEE Trans. Autom. Control 1987, 32, 166-170.

26. Tsinias, J. Further results on the observer design problem. Syst. Control Lett. 1990, 14, 411-418.

27. Ciccarella, G.; Dalla Mora, M.; Germani, A. A Luenberger-like observer for nonlinear systems. Int. J. Control 1994, 57, 537-556.

28. Açikmese, A.; Corless, M. Observers for systems with nonlinearities satisfying incremental quadratic constraints. Automatica 2011, 47, 1339-1348.

29. Djeddi, A.; Dib, D.; Azar, A.T.; Abdelmalek, S. Fractional order unknown inputs fuzzy observer for Takagi-Sugeno systems with unmeasurable premise variables. Mathematics 2019, 7, 984. [CrossRef] 
30. Gauthier, J.; Kupka, I. Deterministic Observation Theory and Applications; Cambridge University Press: Cambridge, UK, 2001.

31. Khalil, H.; Praly, L. High-gain observers in nonlinear feedback control. Int. J. Robust Nonlinear Control 2014, 24, 993-1015.

32. Alessandri, A.; Rossi, A. Increasing-gain observers for nonlinear systems: Stability and design. Automatica 2015, 57, 180-188.

33. Astolfi, D.; Marconi, L. A high-gain nonlinear observer with limited gain power. IEEE Trans. Autom. Control 2015, 60, 3059-3064.

34. Astolfi, D.; Marconi, L.; Praly, L.; Teel, A. Low-power peaking-free high-gain observers. Automatica 2018, 98, 169-179.

35. Imura, J.I.; Sugie, T.; Yoshikawa, T. A Hamilton-Jacobi inequality approach to the strict $H_{\infty}$ control problem of nonlinear systems. Automatica 1996, 32, 645-650.

36. Krichman, M.; Sontag, E.; Wang, Y. Input-output-to-state stability. SIAM J. Control Optim. 2001, 39, $1874-1928$.

37. Rapaport, A.; Astolfi, A. Practical $L_{2}$ disturbance attenuation for nonlinear systems. Automatica 2002, 38, 139-145.

38. Lasserre, J. Global optimization with polynomials and the problem of moments. SIAM J. Optim. 2001, 11, 796-817.

39. Lasserre, J. An Introduction to Polynomial and Semi-Algebraic Optimization; Cambridge University Press: Cambridge, UK, 2015.

40. Grüne, L.; Sontag, E.; Wirth, F. Asymptotic stability equals exponential stability, and ISS equals finite energy gain-If you twist your eyes. Syst. Control Lett. 1999, 38, 127-134.

41. Ahmadi, A.A.; Krstic, M.; Parrilo, P.A. A globally asymptotically stable polynomial vector field with no polynomial Lyapunov function. In Proceedings of the 50th IEEE Conference on Decision and Control, Orlando, FL, USA, 12-15 December 2011; pp. 7579-7580.

42. Blekherman, G.; Parrilo, P.; Thomas, R. Semidefinite Optimization and Convex Algebraic Geometry; SIAM: Philadelphia, PA, USA, 2013.

43. Lasserre, J. Moments, Positive Polynomials and Their Applications; Imperial College Press: London, UK, 2010.

44. Papachristodoulou, A.; Anderson, J.; Valmorbida, G.; Prajna, S.; Seiler, P.; Parrilo, P. SOSTOOLS: Sum of Squares Optimization Toolbox for MATLAB; Version 3.03; California Institute of Technology: Pasadena, CA, USA, 2020.

45. Boyd, S.; El Ghaoui, L.; Feron, E.; Balakrishnan, V. Linear Matrix Inequalities in System and Control Theory; Studies in Applied Mathematics; SIAM: Philadelphia, PA, USA, 1994; Volume 15.

46. Löfberg, J. YALMIP: A Toolbox for Modeling and Optimization in MATLAB. In Proceedings of the CACSD Conference, New Orleans, LA, USA, 2-4 September 2004; pp. 284-289.

47. Casadei, G.; Astolfi, D.; Alessandri, A.; Zaccarian, L. Synchronization in networks of identical nonlinear nystems via nynamic dead zones. IEEE Control Syst. Lett. 2019, 3, 667-672.

48. Astolfi, D.; Postoyan, R.; Nešić, D. Uniting observers. IEEE Trans. Autom. Control 2020, to appear.

49. Alessandri, A.; Gaggero, M. Fast moving horizon state estimation for discrete-time systems using single and multi iteration descent methods. IEEE Trans. Autom. Control 2017, 62, 4499-4511.

(c) 2020 by the author. Licensee MDPI, Basel, Switzerland. This article is an open access article distributed under the terms and conditions of the Creative Commons Attribution (CC BY) license (http:// creativecommons.org/licenses/by/4.0/). 